\title{
IL-37 relieves allergic inflammation by inhibiting the CCL11 signaling pathway in a mouse model of allergic rhinitis
}

\author{
HUIJIA LEI, YIQING SUN and SHIMING QUAN \\ Department of Otorhinolaryngology, Beijing Jishuitan Hospital, Beijing 100035, P.R. China
}

Received April 28, 2019; Accepted June 24, 2020

DOI: $10.3892 /$ etm.2020.9078

\begin{abstract}
Allergic rhinitis (AR) is the allergic inflammation of immune cells in the nasal mucosa, caused by an abnormal T-cell response. Interleukin (IL)-37, a unique member of the IL-1 family with broad anti-inflammatory roles in various autoimmune diseases, participates in the immune regulation of AR. However, the regulatory mechanism of IL-37 in AR has remained elusive. In the present study, a mouse model of AR was established by treating mice with ovalbumin (OVA). Following systemic administration of IL-37, the effects of the cytokine on allergic symptoms were evaluated. The nasal mucosal infiltration of eosinophils was assessed by histopathological observation. The serum and nasal lavage fluid concentrations of immunoglobulin $(\mathrm{Ig}) \mathrm{E}, \mathrm{IgG} 1, \mathrm{IgG} 2 \mathrm{a}$, interferon (IFN)- $\gamma$, IL-4, IL-13, IL-17a and C-C motif cytokine ligand (CCL)11 were determined by ELISA. Treatment with OVA resulted in allergic symptoms, including enhanced eosinophil infiltration in the nasal mucosa, increased thickness of the nasal mucosa and increased levels of $\operatorname{IgE}$, IgG1, IgG2a, IL-4, IL-13, IL-17a and CCL11, but the level of IFN- $\gamma$ was indicated to decrease. After IL-37 treatment, the frequency of nasal rubbing and sneezing was reduced compared with that in the OVA group. IL-37 administration also decreased the number of eosinophils in the nasal mucosa and the thickness of the nasal mucosa, as well as the serum and nasal lavage fluid levels of IgE, IgG1, IgG2a, IL-4, IL-13, IL-17a and CCL11, but the level of IFN- $\gamma$ decreased. In addition, the OVA-induced increases in histamine and substance P levels were reversed by IL-37 administration. CCL11 expression levels were correlated with the expression levels of IFN- $\gamma$, IL-4, IL-13, IL-17a, histamine and substance P. In conclusion, IL-37 alleviated the OVA-induced allergic symptoms and allergic inflammatory response by reducing the serum cytokine levels via decreasing CCL11 expression levels in mice.
\end{abstract}

Correspondence to: Dr Huijia Lei, Department of Otorhinolaryngology, Beijing Jishuitan Hospital, 31 East Xinjiekou Street, Xicheng, Beijing 100035, P.R. China

E-mail: kaixin259@sina.com

Key words: allergic rhinitis, interleukin-37, C-C motif cytokine ligand 11 , mice, $\mathrm{CD} 4^{+} \mathrm{T}$ cells

\section{Introduction}

Allergic rhinitis (AR) is the allergic inflammation of immune cells in the nasal mucosa and is characterized by clinical symptoms and complaints including itching, allergic conjunctivitis, rhinorrhea, nasal congestion and disturbed olfaction (1). AR affects $\sim 10 \%$ of the population worldwide and an increase in the frequency of this disease has been reported (2). AR is closely associated with other inflammatory diseases, including asthma, chronic rhinosinusitis, allergic conjunctivitis and otitis media with effusion and adenoid hypertrophy, which may affect the quality of life of patients with $\operatorname{AR}(3,4)$.

The imbalance of type $2 \mathrm{~T}$ helper (Th2) cells, a subset of $\mathrm{CD}^{+} \mathrm{T}$ cells, has an essential role in the pathogenesis and immunological characteristics of AR (5). A previous study reported that immunoglobulin (Ig)E produced by Th2 cells binds to IgE receptors on the surface of mast cells to sensitize these cells, resulting in the release of histamine and leukotrienes, which induce an AR attack (6). Interleukin (IL)-4 and IL-13 secreted by activated Th2 cells are recognized as key mediators of the pathogenesis of AR, leading to IgE isotype switch (7). Furthermore, IL-17a and interferon (IFN)- $\gamma$ are inflammatory cytokines produced by $\mathrm{Th} 2$ cells that may induce the expression of other cytokines and mucosal proteins. IL-17a and IFN- $\gamma$ are involved in the development and regulation of AR $(8,9)$. Shirasaki et al $(10)$ reported that $\mathrm{Th} 2$ cells were abundant in the nasal mucosa during AR and low Th2 cell counts relieved AR-associated symptoms, suggesting that Th2 cells may be a potential target for the treatment of AR (10).

IL-37 is a unique member of the IL-1 family with broad anti-inflammatory roles during autoimmune diseases, including systemic lupus erythematosus, psoriasis and asthma (11-13). Several studies have reported that IL-37 alleviates allergic inflammation during AR (14-16). In animal models, administration of recombinant human IL-37 may attenuate the local allergic symptoms of AR and decrease the expression of cytokines produced by Th2 and Th17 cells in the nasal mucosa (15). IL-37 has also been demonstrated to regulate aberrant $\mathrm{T}$-cell immune responses in patients with AR (16).

Wang et al (14) reported that phosphorylation of STAT proteins, together with IL-37 signaling and exogenous IL-37 treatment, is able to attenuate allergic symptoms (nasal rubbing and sneezing) by decreasing STAT6 or STAT3 expression levels in $\mathrm{CD} 4^{+} \mathrm{T}$-cell subsets (14). The increased expression of 
IL-1R8 in $\mathrm{CD}^{+} \mathrm{T}$ cells may be associated with the potential role of IL-37 during AR-mediated anti-inflammation (16). Further studies have focused on the role of IL-37 in limiting innate inflammation primarily by inhibiting the production of proinflammatory cytokines by macrophages or epithelial cells $(17,18)$. However, the underlying anti-inflammatory mechanism of IL-37, as well as its ability to alleviate the allergic symptoms of AR, have yet to be determined.

$\mathrm{C}-\mathrm{C}$ motif cytokine ligand (CCL)11 is critical for attracting eosinophils during allergic asthma in mice (19,20). IL-4/IL-13 signaling may induce CCL11 secretion in Th2 cells (21), and interruption of IL-4 and IL-13 synthesis significantly decreases CCL11 production, which further reduces pulmonary eosinophilia in several asthma models $(22,23)$. In a previous animal study, IL-37 has been reported to inhibit the production of IL-4/IL-13 but induce the production of CCL11, thereby stimulating anti-inflammatory mechanisms in allergic asthma (24). However, the exact mechanisms by which IL-37 inhibits IL-4/IL-13 and induces CCL11 to exert its anti-inflammatory effects in AR have yet to be elucidated.

On the basis of the aforementioned studies, it was hypothesized that IL-37 alleviates the allergic symptoms of AR via CCL11 signaling in Th2 cells. To further understand the role of IL-37 in CCL11 signaling, a mouse model of AR was established by treating animals with ovalbumin (OVA). The allergic symptoms were investigated and the serum and nasal lavage fluid levels of IgE, IgG1, IgG2a, IFN- $\gamma$, IL-4, IL-13, IL-17a and CCL11, as well as histamine and substance P, were determined by ELISA. In addition, the number of eosinophils was determined by histopathological observation.

\section{Materials and methods}

Animals. A total of 40 male BALB/c mice (age, 6-8 weeks; weight, 20-23 g) were purchased from Beijing SPF Vital Laboratory Animal Technology Company. The mice were housed in a limited access rodent facility with 8 rats per polycarbonate cage in a room with a $12 \mathrm{~h}$ light, $12 \mathrm{~h}$ dark cycle (lights on from 7:00 a.m. to 7:00 p.m.), with a relative humidity maintained at $55 \pm 15 \%$, and a constant temperature $\left(24 \pm 2^{\circ} \mathrm{C}\right)$. The experiments were performed in accordance with the National Institutes of Health Guidelines for the Care and Use of Laboratory Animals and approved by the Animal Care and Use Committee of Beijing Jishuitan Hospital (Beijing, China).

Experimental procedure. To induce sensitization, mice were administered with an intraperitoneal injection of $1 \mathrm{mg} / \mathrm{ml}$ OVA (Grade V; Sigma-Aldrich; Merck KGaA) and $20 \mathrm{mg} / \mathrm{ml}$ aluminum hydroxide (Sigma-Aldrich; Merck KGaA) in sterile saline $(0.1 \mathrm{ml} /$ mouse) on days $1,3,5,7,9,11$ and 13 . To induce AR, mice were subsequently intranasally administered $60 \mathrm{mg} / \mathrm{ml}$ OVA in sterile saline $(20 \mu \mathrm{l} / \mathrm{mouse})$ from day 20 to day $30(14)$. The control group $(n=10)$ did not receive any treatment.

Experimental design and pharmacological treatment. The experimental design is presented in Fig. 1. OVA-exposed mice were randomly assigned to one of the following three groups ( $n=10$ per group): The OVA group received no treatment; the OVA + Sal group received $1 \mu \mathrm{g}$ saline by intraperitoneal injection prior to each OVA challenge and for 5 days between day 26 and day 30; and the OVA + IL-37 group received $1 \mu \mathrm{g}$ recombinant human IL-37 (dissolved in $50 \mu 1$ sterile PBS, R\&D Systems, Inc.) by intraperitoneal injection prior to each OVA challenge and for 5 days between day 26 and day 30 according to a previous protocol (14).

Evaluation of allergic symptoms. Nasal symptoms were evaluated by counting the frequency of nasal rubbing and sneezing (25). The frequency of nasal rubbing and sneezing within a 15 -min period after the final allergen challenge was recorded by two observers blinded to the experimental conditions.

Collection of serum and nasal lavage fluid and ELISA. At $24 \mathrm{~h}$ after the last administration of OVA, the mice were anesthetized with isoflurane. Cardiac blood samples were harvested from each mouse in EDTA-coated tubes, maintained on ice, centrifuged at $4,000 \times \mathrm{g}$ for $10 \mathrm{~min}$ at $4^{\circ} \mathrm{C}$ and subsequently, serum was isolated. ELISA was performed to determine the following: Total IgE (Mouse IgE ELISA kit; cat. no. SEKM-0095; Beijing Solarbio Science \& Technology Co., Ltd.), IgG1 (Mouse IgG1 ELISA kit; cat. no. SEKM-0097; Beijing Solarbio Science \& Technology Co., Ltd.), IgG2a (Mouse IgG2a ELISA kit; cat. no. SEKM-0099; Beijing Solarbio Science \& Technology Co., Ltd.), IFN- $\gamma$ (Mouse IFN- $\gamma$ ELISA kit; cat. no. PI508; Beyotime Institute of Biotechnology), IL-4 (Mouse IL-4 ELISA kit; cat. no. PI612; Beyotime Institute of Biotechnology), IL-13 (Mouse IL-13 PicoKine ELISA kit; cat. no. EK0425; Boster Biological Technology), IL-17a (Mouse IL-17a ELISA kit; cat. no. PI545; Beyotime Institute of Biotechnology) and CCL11 (Mouse CCL11 PicoKine ELISA kit; cat. no. EK0330; Boster Biological Technology). Concentrations were determined by ELISA using specific rabbit polyclonal antibodies (Abcam) according to the manufacturer's protocol.

After the collection of blood, the mice were sacrificed with an overdose of isoflurane. When the cardiac activity and respiration ceased 15 min later, nasal lavage fluid was collected using an 18-gauge catheter. In brief, the trachea was partly resected, a catheter was inserted from the trachea into the nasopharynx and the nasal passages were gently perfused with $1 \mathrm{ml}$ saline. The nasal lavage fluid was centrifuged at $10,000 \mathrm{x} \mathrm{g}$ for $10 \mathrm{~min}$ at $4^{\circ} \mathrm{C}$. The concentrations of IFN- $\gamma$, IL-4, IL-13, IL-17a and CCL11 were determined by ELISA using the aforementioned kits. In addition, histamine (Histamine ELISA kit; cat. no. ab213975; Abcam) and substance P (Substance P ELISA kit; cat. no. ab133029; Abcam) were measured by ELISA.

Histopathologic observation. After $24 \mathrm{~h}$ of the last challenge on day 30 , a portion of the nasal mucosa was excised and fixed in $10 \%$ formalin for 3 days at room temperature. The tissues were cut into $4-\mu \mathrm{m}$ sections and stained for $1 \mathrm{~min}$ at room temperature using a haematoxylin-eosin staining kit (Beyotime Institute of Biotechnology) to assess the extent of inflammatory cell infiltration. A total of 5 random fields of vision in the nasal mucosal sections were examined using an Olympus FluoView 1200 confocal microscope (Olympus Corp.) and photomicrographs (magnification, x400) of representative nasal mucosa areas were acquired. The thickness of the nasal 


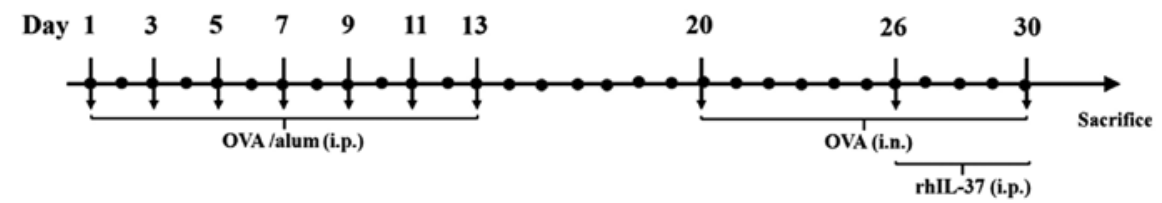

Figure 1. Experimental design of the mouse model of AR. To induce sensitization, mice were administered OVA and alum i.p. on days 1,3,5, 7,9,11 and 13. To induce AR, the mice were challenged i.n. with OVA from day 20 to 30 and were subsequently administered rhIL-37 i.p. for 5 consecutive days from day 26 to 30 . AR, allergic rhinitis; OVA, ovalbumin; alum, aluminum hydroxide; i.p., intraperitoneally; i.n., intranasally; rhIL-37, recombinant human interleukin 37.
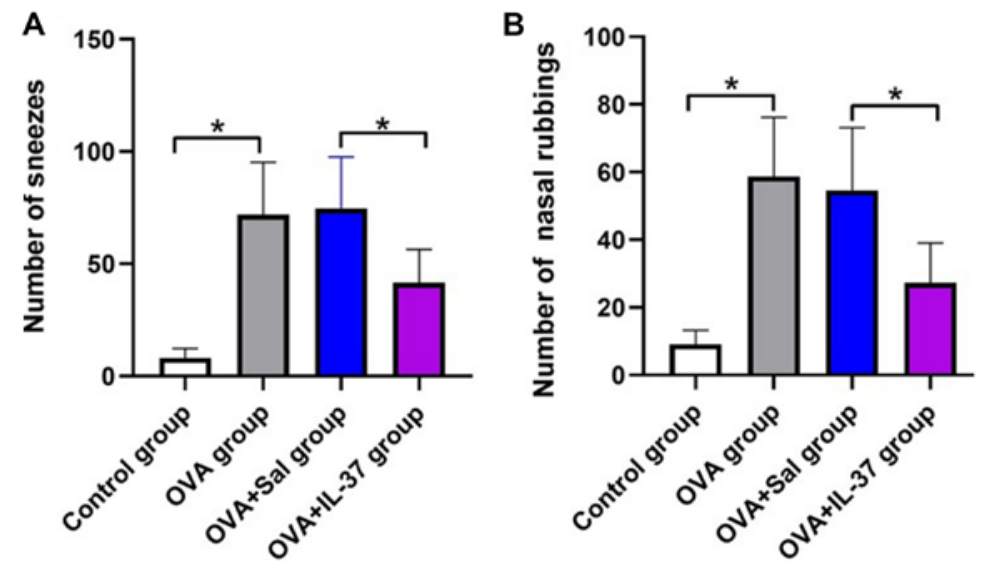

Figure 2. Systemic administration of IL-37 attenuates OVA-induced allergic symptoms. Frequency of (A) sneezing and (B) nasal rubbing. Values are expressed as the mean \pm standard deviation $(n=40)$. "P<0.05, as indicated. IL, interleukin; OVA, ovalbumin; Sal, saline.

mucosa and the number of eosinophils were calculated using Image J software (Version 1.5.1; National Institutes of Health)

Statistical analysis. Values are expressed as the mean \pm standard deviation. Statistical analyses were performed using SPSS software (version 23.0; IBM Corp.) and GraphPad Prism software (version 7.0; GraphPad Software, Inc.). One-way analysis of variance followed by Bonferroni's correction was used for multiple comparisons among four groups. The correlation between two variables was assessed by Pearson correlation analysis. $\mathrm{P}<0.05$ was considered to indicate a statistically significant difference.

\section{Results}

Allergic symptoms. The effects of IL-37 administration on the allergic symptoms of the mice with OVA-induced AR are presented in Fig. 2. The frequency of nasal rubbing $(\mathrm{P}<0.001)$ and sneezing $(\mathrm{P}<0.05)$ in the OVA group was significantly increased compared with that in the control group (Fig. 2A and B). After IL-37 administration, the frequency of nasal rubbing $(\mathrm{P}<0.001)$ and sneezing $(\mathrm{P}<0.05)$ in the OVA + IL-37 group was significantly decreased compared with that in the OVA + Sal group (Fig. 2A and B).

Serum IgE, IgG1 and IgG2a levels. Serum IgE, IgG1 and IgG2a levels in mice were measured by ELISA and the results are presented in Fig. 3. IgE levels in the serum were significantly higher in the OVA group compared with those in the control group $(\mathrm{P}<0.001$; Fig. 3A). Treatment with IL-37 significantly reduced the IgE levels in the serum of OVA-treated mice $(\mathrm{P}<0.001$; Fig. 3A). IgG1 levels in the OVA group were significantly increased compared with those in the control group. However, IgG1 serum levels were decreased in the OVA + IL-37 group compared with the OVA group $(\mathrm{P}<0.001$; Fig. 3B). Furthermore, IgG2a serum levels were significantly decreased in the OVA group compared with those in the control group, but this effect was reversed by IL-37 treatment $(\mathrm{P}<0.001$; Fig. 3C).

Thickness of the nasal mucosa and number of eosinophils. The thickness of the nasal mucosa and number of eosinophils were histologically determined (Fig. 4). Representative histological images for the different groups are provided in Fig. 4A, B, D and E. The thickness of the nasal mucosa was significantly increased in the OVA group compared with that in the control group $(\mathrm{P}<0.001$; Fig. 4C). Following IL-37 treatment, a significant decrease in the thickness of the nasal mucosa of OVA-treated mice was observed ( $\mathrm{P}<0.001$; Fig. 4C). The eosinophil count in the nasal mucosa was significantly increased in the OVA group compared with that in the control group $(\mathrm{P}<0.001$; Fig. 4F), and similarly, following IL-37 treatment, OVA-treated mice displayed a significant decrease in the eosinophil count $(\mathrm{P}<0.001 ;$ Fig. $4 \mathrm{~F})$.

Nasal lavage fluid cytokine or chemical mediator levels. As presented in Fig. 5, the levels of cytokines and chemical mediators, including IL-4 (Fig. 5B), IL-13 (Fig. 5C), IL-17a (Fig. 5D), CCL11 (Fig. 5E), histamine (Fig. 5F) and substance $\mathrm{P}$ (Fig. 5G), were significantly increased in the nasal lavage fluid of the OVA group compared with those in the control group. However, IFN- $\gamma$ levels were decreased in the nasal lavage fluid of the OVA group compared with those in the control group $(\mathrm{P}<0.01$; Fig. 5A). After IL-37 treatment, the effects of OVA 

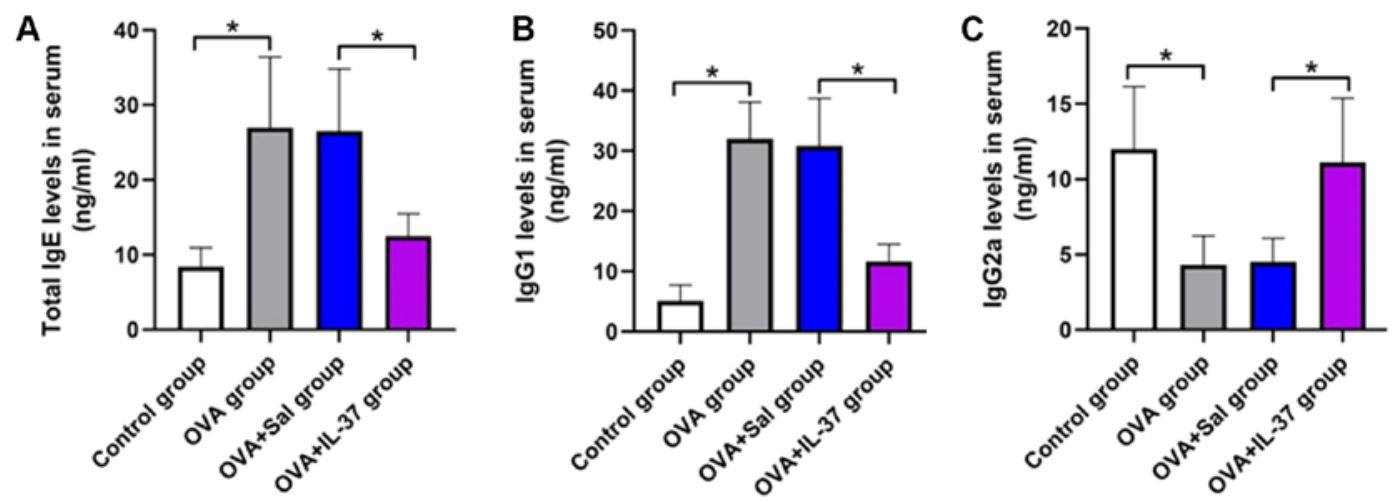

Figure 3. Systemic administration of IL-37 reduces the OVA-induced serum levels of IgE, IgG1 and IgG2a in a mouse model of allergic rhinitis. Serum levels of (A) $\operatorname{IgE},(B) \operatorname{IgG1}$ and (C) IgG2a. Values are expressed as the mean \pm standard deviation ( $\mathrm{n}=40)$. " $\mathrm{P}<0.05$, as indicated. IL, interleukin; OVA, ovalbumin; Ig, immunoglobulin; Sal, saline.
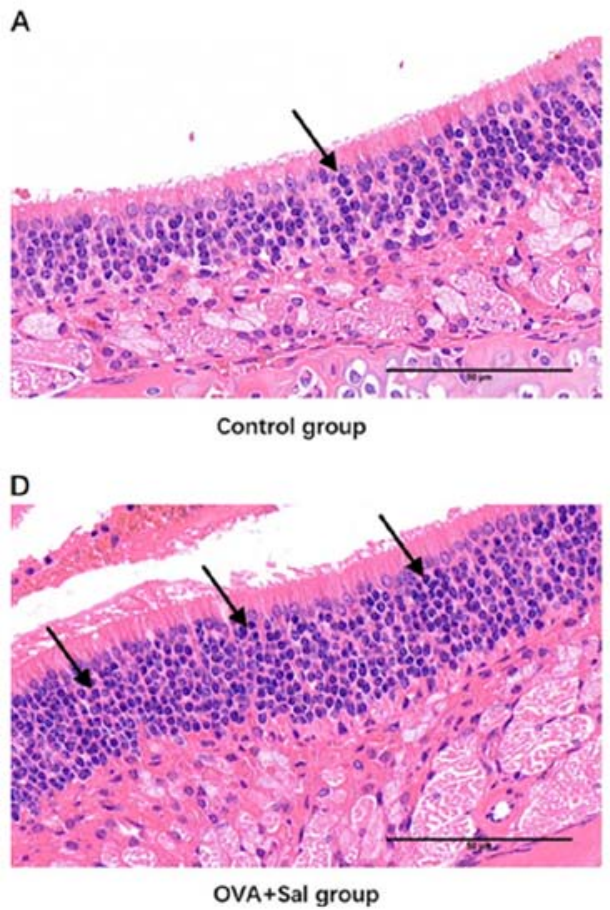

E
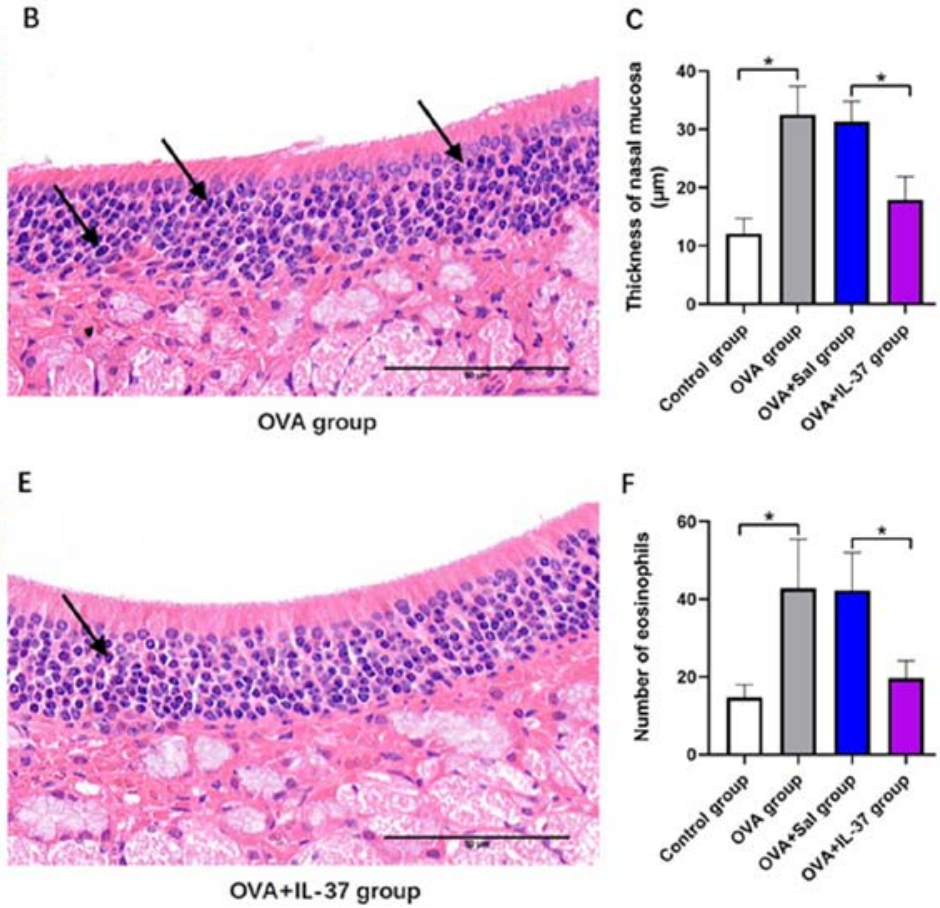

$\mathrm{F}$

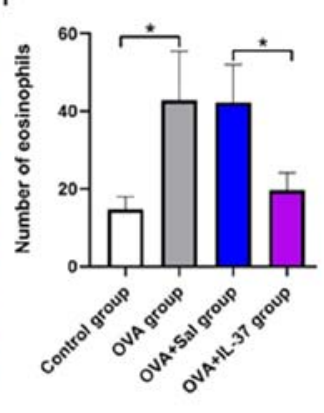

Figure 4. Systemic administration of IL-37 attenuates OVA-induced nasal eosinophil infiltration and alterations to the thickness of the nasal mucosa in a mouse model of allergic rhinitis. Representative photomicrographs of the nasal mucosa in the (A) control, (B) OVA, (C) OVA + Sal and (D) OVA + IL-37 groups. The nasal mucosa tissues were stained with haematoxylin \& eosin and histopathologically observed (magnification, $\mathrm{x} 400$; scale bar, $50 \mu \mathrm{m}$ ). (E) Eosinophil count and $(\mathrm{F})$ thickness of the nasal mucosa. Values are expressed as the standard deviation $(\mathrm{n}=40)$. The black arrows represent eosinophils. ${ }^{*} \mathrm{P}<0.05$, as indicated. IL, interleukin; OVA, ovalbumin; Sal, saline.

on the levels of the abovementioned cytokines and chemical mediators in the nasal lavage fluid were significantly inhibited or even reversed $(\mathrm{P}<0.001$; Fig. $5 \mathrm{~A}-\mathrm{F})$. A positive correlation between the levels of CCL11 and IL-4 $\left(\mathrm{r}^{2}=0.6765 ; \mathrm{P}<0.05\right.$; Fig. 5I), IL-13 ( $r^{2}=0.8650$; $P<0.05$; Fig. 5J), IL-17a $\left(r^{2}=0.4562\right.$; $\mathrm{P}<0.05$; Fig. 5K), histamine $\left(\mathrm{r}^{2}=0.8439\right.$; $\mathrm{P}<0.05$; Fig. 5L) and substance $\mathrm{P}\left(\mathrm{r}^{2}=0.8406 ; \mathrm{P}<0.05\right.$; Fig. $\left.5 \mathrm{M}\right)$ was identified. Furthermore, a negative correlation between the levels of CCL11 and IFN- $\gamma$ was obtained $\left(r^{2}=0.6641\right.$; $<<0.05$; Fig. $\left.5 \mathrm{H}\right)$.

Serum cytokine levels. The serum cytokine levels are presented in Fig. 6. The serum levels of IL-4 (Fig. 6B), IL-13 (Fig. 6C), IL-17a (Fig. 6D) and CCL11 (Fig. 6E) were significantly increased, while the levels of IFN- $\gamma$ were significantly decreased (Fig. 6A) in the OVA group compared with those in the control group $(\mathrm{P}<0.001)$. Following IL-37 treatment, the effect of OVA on the serum levels of each cytokine was significantly inhibited or reversed $(\mathrm{P}<0.001$; Fig. 6A-E). A positive correlation between the serum levels of CCL11 and IL-4 ( $r^{2}=0.6817 ; \mathrm{P}<0.05$; Fig. 6G), IL-13 ( $\mathrm{r}^{2}=0.6955 ; \mathrm{P}<0.05$; Fig. $6 \mathrm{H})$ and IL-17a $\left(\mathrm{r}^{2}=0.2384 ; \mathrm{P}<0.05\right.$; Fig. $\left.6 \mathrm{I}\right)$ was identified. By contrast, a negative correlation between the serum levels of CCL11 and IFN- $\gamma\left(r^{2}=0.4294 ; \mathrm{P}<0.05\right.$; Fig. $\left.6 \mathrm{~F}\right)$ was obtained.

\section{Discussion}

In the present study, the protective effect and ability of IL-37 to attenuate the allergic inflammatory response by decreasing the 
A

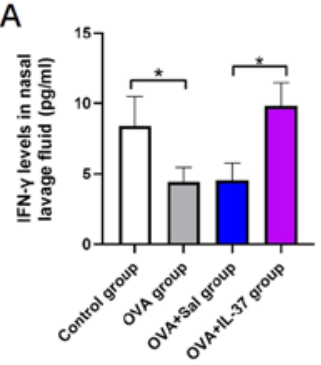

F

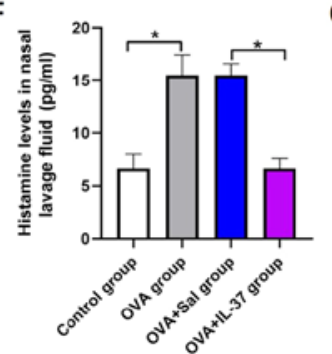

J

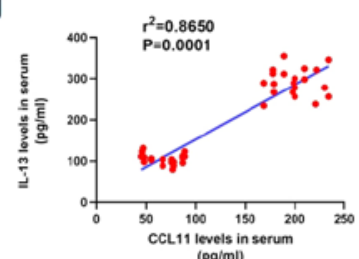

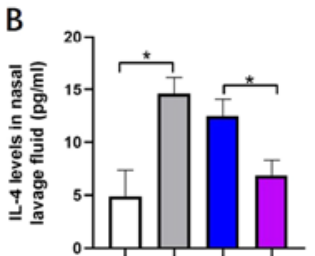

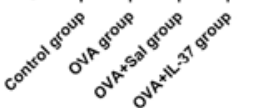

G

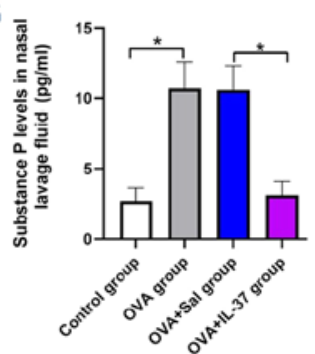

K

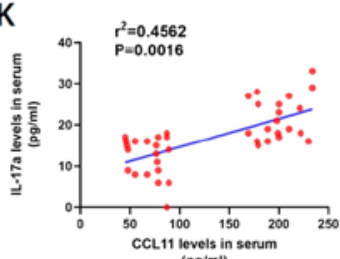

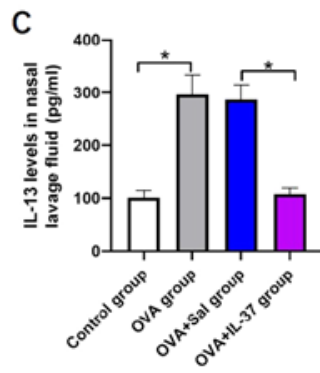

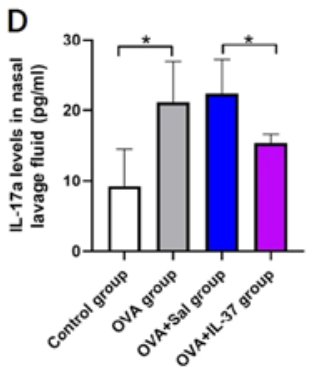

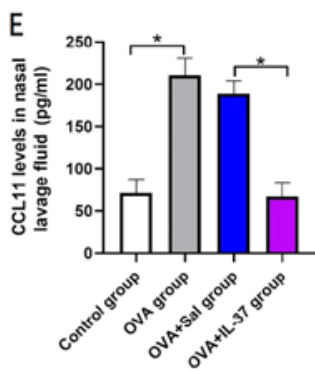

I
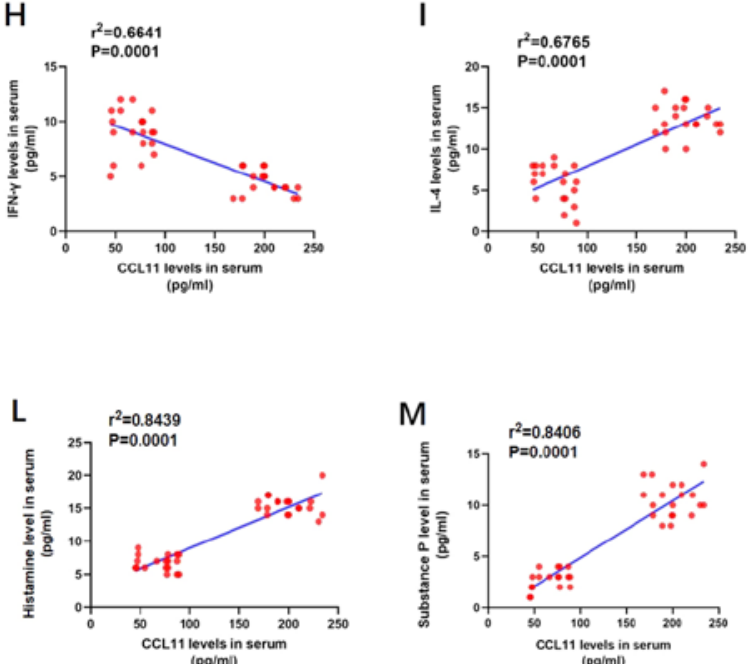

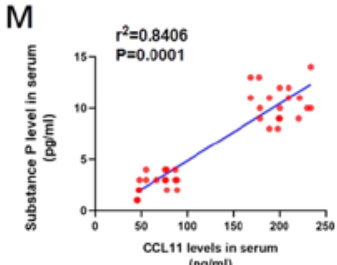

Figure 5. Systemic administration of IL-37 reverses OVA-induced effects on the levels of cytokines or chemical mediators in the nasal lavage fluid in a mouse model of allergic rhinitis, and the correlation between CCL11 and IFN- $\gamma$, IL-4, IL-13, IL-17a, histamine and substance P. Nasal lavage fluid level of (A) IFN- $\gamma$, (B) IL-4, (C) IL-13, (D) IL-17a, (E) CCL11, (F) histamine and (G) substance P. The correlation between nasal lavage fluid levels of CCL11 and (H) IFN- $\gamma$, (I) IL-4, (J) IL-13, (K) IL-17a, (L) Histamine and (M) Substance. P Values are expressed as the mean \pm standard deviation (n=40). ${ }^{\mathrm{P}<0.05}$, as indicated. IL, interleukin; OVA, ovalbumin; CCL11, C-C motif cytokine ligand 11 ; IFN- $\gamma$, interferon- $\gamma$; Sal, saline.
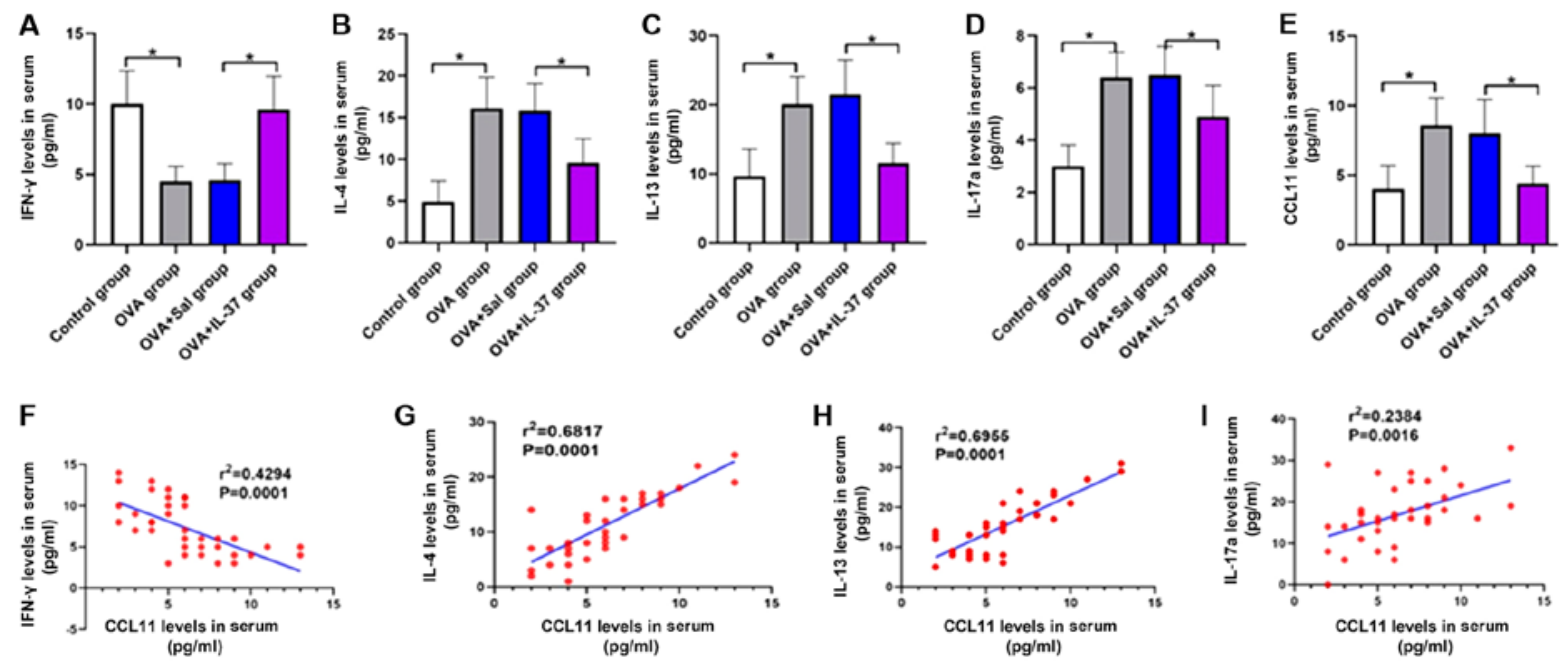

Figure 6. Systemic administration of IL-37 reverses the OVA-induced effects on serum levels of IFN- $\gamma$, IL-4, IL-13, IL-17a and CCL11 in a mouse model of allergic rhinitis, and the correlation between serum levels of CCL11 and IFN- $\gamma$, IL-4, IL-13 and IL-17a. Serum levels of (A) IFN- $\gamma$, (B) IL-4, (C) IL-13, (D) IL-17a and (E) CCL11. The correlation between serum levels of CCL11 and (F) IFN- $\gamma$, (G) IL-4, (H) IL-13 and (I) IL-17a. Values are expressed as the mean \pm standard deviation $(\mathrm{n}=40)$. "P<0.05, as indicated. IL, interleukin; OVA, ovalbumin; IFN- $\gamma$, interferon- $\gamma$; CCL11, C-C motif cytokine ligand 11; Sal, saline.

production of CCL11 in a mouse model of OVA-induced AR was investigated. Allergic symptoms, including nasal mucosal eosinophilia, increased thickness of the nasal mucosa, as well as altered serum IgE, IgG1 and Ig G2a levels, were reduced or reversed in AR mice treated with IL-37. Furthermore, IL-37 decreased the levels of cytokines, including IL-4, IL-13, IL-17a and CCL11, in the serum and nasal lavage fluid of mice with OVA-induced AR. However, the level of IFN- $\gamma$ in the serum 
and nasal lavage fluid of mice could be increased by IL-37 treatment. In addition, OVA-induced increases in the levels of histamine and substance P were reversed by IL-37 administration. CCL11 levels displayed a positive correlation with the levels of IL-4, IL-13, IL-17a, histamine and substance P. CCL11 displayed a negative correlation with the expression of IFN- $\gamma$, which suggested that IL-37 may attenuate the allergic inflammatory response by decreasing CCL11 expression in an OVA-induced mouse model of AR.

Immunoglobulins have major roles in mediating allergic and inflammatory reactions. The expression of several immunoglobulin antibodies, including $\mathrm{IgE}, \mathrm{IgG} 1$ and $\mathrm{IgG} 2 \mathrm{a}$, has been reported to be involved in $\mathrm{B}$-cell immune responses controlled by cytokines produced by Th cells, such as TNF- $\alpha$, IL-4, IL-5, IL-10, IL-12 and IL-13 (1,26). In particular, allergens may activate the binding of $\operatorname{IgE}$ to the high-affinity $\operatorname{IgE}$ receptor FceRI on the surface of eosinophils. Subsequently, chemical mediators are released into the surrounding tissues, causing allergic symptoms $(2,27)$. In the present study, the altered serum profiles of IgE, IgG1 and Ig G2a in OVA-treated mice were reversed by IL-37 treatment, suggesting that IL-37 may downregulate immune responses in $\mathrm{CD} 4^{+} \mathrm{T}$ cells. Following IL-37 treatment, the OVA group displayed significantly reduced allergic symptoms (nasal rubbing and sneezing). The results suggested that systemic administration of IL-37 decreased the hallmarks of experimental AR by decreasing the production of $\mathrm{IgE}, \mathrm{IgG} 1$ and $\mathrm{IgG} 2 \mathrm{a}$.

The infiltration of eosinophils into the mucosa and the thickness of the nasal mucosa are important pathological features of AR. Limited chronic inflammation and a priming effect are stimulated by eosinophil infiltration into the nasal mucosa, both of which may intensify nasal hyperreactivity (28). In the present study, histopathological observations indicated a high degree of eosinophil infiltration in the interstitium of the nasal mucosa and an increase in the thickness of the nasal mucosa in the mouse model of AR. Furthermore, the degree of eosinophil infiltration and the thickness of the nasal mucosa were reduced by IL-37, which is consistent with the results of a study performed by Shahsavan et al (29). These results indicated that IL-37 may attenuate the allergic inflammatory response by decreasing eosinophil infiltration in the interstitium of the nasal mucosa.

IL-4 and IL-13 are immunoregulatory cytokines secreted predominantly by activated Th2 cells and serve as key mediators of the allergic response, including the induction of isotype switching to $\operatorname{IgE}$ and the promotion of eosinophil migration across the endothelium of Th2 lymphocytes $(30,31)$. Increasing evidence suggests that Th2 cytokines, including IL-4 and IL-13, which are downregulated by $\mathrm{T}$ cells, are elevated in patients with AR, inducing the itch response via signaling in sensory neurons $(32,33)$. IL-4 and IL-13 are secreted primarily by eosinophils and basophils, and they bind to the IgE receptor with high affinity to initiate IgE-dependent inflammatory reactions and IgE production by B cells (34). Furthermore, IL-4 may induce polyamine synthesis, which exerts anti-inflammatory effects (35). In the present study, IL-37 decreased the IL-4 and IL-13 levels in the serum and nasal lavage fluid, indicating that IL-37 attenuated the allergic inflammatory response by downregulating IL-4 and IL-13 expression. Huang et al (36) reported that IL-37 is able to downregulate IL-4/IL-13 expression by inhibiting STAT6 activation and STAT3 phosphorylation. The results also suggested a potential underlying mechanism of IL-37, indicating that IL-37 reversed the serum profile of $\operatorname{IgE}$ and eosinophil infiltration in the mucosa.

IL-17 is involved in airway hyperreactivity and mucus hypersecretion in the upper airway during AR (37). After OVA administration, the IL-17 level was significantly increased, which is consistent with the results of a previous study (1). By contrast, IL-37 administration reversed the OVA-induced increases in the serum and nasal lavage fluid levels of IL-17, indicating that IL-37 may reverse allergic symptoms (nasal rubbing and sneezing). IFN- $\gamma$ is the principal Th1 effector cytokine, which affects Th1/Th2 differentiation, triggers macrophage production and inhibits Th2-cell proliferation (38). Zhu et al (39) reported that reductions in IFN- $\gamma$ release in in vitro co-culture with IL-37 may partly rely on the suppressed transcriptional activity of NF- $\mathrm{NB}$ (39). The present study suggested that the concentration of IFN- $\gamma$ was increased in the OVA + IL-37 group compared with that in the OVA group, further indicating that IL-37 shifted the Th2 immune response to allergens to a Th1 immune response.

Although the levels of IL-4, IL-13 and IL-17 were significantly reduced by IL-37, similar to the results reported in previous studies $(14,16,24,39)$, the mechanism of IL-37 in the regulation of cytokines has yet to be determined. CCL11 is critical for the attraction of eosinophils during allergic asthma. Therefore, the present study investigated the serum and nasal lavage fluid levels of CCL11. CCL11 levels were significantly increased in the OVA group compared with those in the control group and were reduced following treatment with IL-37. Of note, CCL11 displayed a positive correlation with various cytokines, including IL-4, IL-13 and IL-17. According to the literature, the inhibitory effect of IL-37 on IL-4/IL-13 is mediated by a reduction in CCL11 production in house dust mite-induced allergic asthma (24). Therefore, IL-37-mediated alterations to the levels of CCL11 may have a role in the regulation of cytokines, including IFN- $\gamma$, IL-4, IL-13 and IL-17.

Despite the important role of IL-37 in the regulation of CCL11-mediated OVA-induced AR, IL-37 has previously been reported to inhibit STAT6 activation and STAT3 phosphorylation to alleviate pulmonary eosinophilia and airway remodeling (36). The possible mechanisms by which IL-37 regulates CCL11 and CCL11 subsequently regulates serum cytokine levels were not identified in the present study and further investigation is thus required. In addition, the IL-2 receptor has been implicated in immune responses and may be a potential mechanism by which IL-37 relieves allergic inflammation (40). Collectively, the results of the present study along with those of previous studies suggest that further investigation is required to identify the underlying mechanisms of IL-37 during AR.

The present study reported novel results on the effect of IL-37 in a mouse model of OVA-induced AR. The results suggested that IL-37 reversed allergic symptoms and the allergic inflammatory response by downregulating serum cytokine levels and inhibiting CCL11 expression.

\section{Acknowledgements}

Not applicable. 


\section{Funding}

No funding was received.

\section{Availability of data and materials}

The datasets used and/or analyzed during the current study are available from the corresponding author on reasonable request.

\section{Authors' contributions}

HL conceived the idea and designed the study. HL, YS and SQ performed the experiments. HL and YS analyzed the data and performed the statistical analysis. HL wrote the manuscript. All authors have reviewed the manuscript and read and approved the final manuscript.

\section{Ethics approval and consent to participate}

The present study was approved by the Animal Care and Use Committee of Beijing Jishuitan Hospital (Beijing, China).

\section{Patient consent for publication}

Not applicable.

\section{Competing interests}

The authors declare that they have no competing interests.

\section{References}

1. Fan Y, Piao CH, Hyeon E, Jung SY, Eom JE, Shin HS, Song CH and Chai OH: Gallic acid alleviates nasal inflammation via activation of Th1 and inhibition of Th2 and Th17 in a mouse model of allergic rhinitis. Int Immunopharmacol 70: 512-519, 2019.

2. Skoner DP: Allergic rhinitis: Definition, epidemiology, pathophysiology, detection, and diagnosis. J Allergy Clin Immunol 108 (Suppl): S2-S8, 2001.

3. Meltzer EO, Blaiss MS, Derebery MJ, Mahr TA, Gordon BR, Sheth KK, Simmons AL, Wingertzahn MA and Boyle JM: Burden of allergic rhinitis: Results from the Pediatric Allergies in America survey. J Allergy Clin Immunol 124 (Suppl): S43-S70, 2009.

4. Sritipsukho P, Satdhabudha A and Nanthapisal S: Effect of allergic rhinitis and asthma on the quality of life in young Thai adolescents. Asian Pac J Allergy Immunol 33: 222-226, 2015.

5. Pilette C, Jacobson MR, Ratajczak C, Detry B, Banfield G, VanSnick J, Durham SR and Nouri-Aria KT: Aberrant dendritic cell function conditions Th2-cell polarization in allergic rhinitis. Allergy 68: 312-321, 2013.

6. Ciprandi G, Marseglia GL, Castagnoli R, Valsecchi C, Tagliacarne C, Caimmi S and Licari A: From IgE to clinical trials of allergic rhinitis. Expert Rev Clin Immunol 11: 1321-1333, 2015.

7. Akdis $M$ and Akdis CA: Mechanisms of allergen-specific immunotherapy: Multiple suppressor factors at work in immune tolerance to allergens. J Allergy Clin Immunol 133: 621-631, 2014

8. Wang M, Zhang W, Shang J, Yang J, Zhang L and Bachert C: Immunomodulatory effects of IL-23 and IL-17 in a mouse model of allergic rhinitis. Clin Exp Allergy 43: 956-966, 2013.

9. Moon IJ, Hong SL, Kim DY, Lee CH, Rhee CS and Min YG: Blocking interleukin-17 attenuates enhanced inflammation by staphylococcal enterotoxin B in murine allergic rhinitis model. Acta Otolaryngol 132 (Suppl 1): S6-S12, 2012.

10. Shirasaki H, Kanaizumi E, Seki N and Himi T: Correlation of Local FOXP3-Expressing T Cells and Th1-Th2 Balance in Perennial Allergic Nasal Mucosa. Int J Otolaryngol 2011: 259867, 2011.
11. Boraschi D, Lucchesi D, Hainzl S, Leitner M, Maier E, Mangelberger D, Oostingh GJ, Pfaller T, Pixner C, Posselt G, et al: IL-37: A new anti-inflammatory cytokine of the IL-1 family. Eur Cytokine Netw 22: 127-147, 2011.

12. Ye L, Ji L, Wen Z, Zhou Y, Hu D, Li Y, Yu T, Chen B, Zhang J, Ding L, et al: IL-37 inhibits the production of inflammatory cytokines in peripheral blood mononuclear cells of patients with systemic lupus erythematosus: Its correlation with disease activity. J Transl Med 12: 69, 2014

13. Teng X, Hu Z, Wei X, Wang Z, Guan T, Liu N, Liu X, Ye N, Deng G, Luo C, et al: IL-37 ameliorates the inflammatory process in psoriasis by suppressing proinflammatory cytokine production. J Immunol 192: 1815-1823, 2014.

14. Wang J, Shen Y, Li C, Liu C, Wang ZH, Li YS, Ke X and Hu GH: IL-37 attenuates allergic process via STAT6/STAT3 pathways in murine allergic rhinitis. Int Immunopharmacol 69: 27-33, 2019.

15. Kim DH, Kim SW, Kim SW and Kang JM: Interleukin-37 relieves allergic inflammation in a house dust mite allergic rhinitis murine model. Iran J Allergy Asthma Immunol 16: 404-417, 2017.

16. Li C, Shen Y, Wang J, Ma ZX, Ke X, Wang ZH, Hong SL and $\mathrm{Hu}$ GH: Increased expression of IL-1R8 and a possible immunomodulatory role of its ligand IL-37 in allergic rhinitis patients. Int Immunopharmacol 60: 152-159, 2018.

17. Nold MF, Nold-Petry CA, Zepp JA, Palmer BE, Bufler P and Dinarello CA: IL-37 is a fundamental inhibitor of innate immunity. Nat Immunol 11: 1014-1022, 2010.

18. Nold-Petry CA, Lo CY, Rudloff I, Elgass KD, Li S, Gantier MP, Lotz-Havla AS, Gersting SW, Cho SX, Lao JC, et al: IL-37 requires the receptors IL-18R $\alpha$ and IL-1R8 (SIGIRR) to carry out its multifaceted anti-inflammatory program upon innate signal transduction. Nat Immunol 16: 354-365, 2015.

19. Kim J, Merry AC, Nemzek JA, Bolgos GL, Siddiqui J and Remick DG: Eotaxin represents the principal eosinophil chemoattractant in a novel murine asthma model induced by house dust containing cockroach allergens. J Immunol 167: 2808-2815, 2001.

20. Pope SM, Zimmermann N, Stringer KF, Karow ML and Rothenberg ME: The eotaxin chemokines and CCR3 are fundamental regulators of allergen-induced pulmonary eosinophilia. J Immunol 175: 5341-5350, 2005.

21. Kuperman DA and Schleimer RP: Interleukin-4, interleukin-13, signal transducer and activator of transcription factor 6 , and allergic asthma. Curr Mol Med 8: 384-392, 2008.

22. Hosokawa Y, Hosokawa I, Shindo S, Ozaki K and Matsuo T: IL-4 Modulates CCL11 and CCL20 Productions from IL-1 $\beta$-Stimulated Human Periodontal Ligament Cells. Cell Physiol Biochem 38: 153-159, 2016.

23. Miyagawa Y, Murakami A and Ebihara N: The proteolytic effect of mast cell tryptase to eotaxin-1/CCL11-eotaxin-2/CCL24 and eotaxin-3/CCL26 produced by conjunctival fibroblasts. Jpn J Ophthalmol 63: 215-220, 2019.

24. Lv J, Xiong Y, Li W, Cui X, Cheng X, Leng Q and He R: IL-37 inhibits IL-4/IL-13-induced CCL11 production and lung eosinophilia in murine allergic asthma. Allergy 73: 1642-1652, 2018.

25. Bui TT, Piao CH, Song CH and Chai OH: Skullcapflavone II attenuates ovalbumin-induced allergic rhinitis through the blocking of Th2 cytokine production and mast cell histamine release. Int Immunopharmacol 52: 77-84, 2017.

26. Zhao N, Liu Y, Liang H and Jiang X: Bone marrow-derived mesenchymal stem cells reduce immune reaction in a mouse model of allergic rhinitis. Am J Transl Res 8: 5628-5636, 2016.

27. Humbles AA, Lloyd CM, McMillan SJ, Friend DS, Xanthou G, McKenna EE, Ghiran S, Gerard NP, Yu C, Orkin SH, et al: A critical role for eosinophils in allergic airways remodeling. Science 305: 1776-1779, 2004.

28. Canonica GW and Compalati E: Minimal persistent inflammation in allergic rhinitis: Implications for current treatment strategies. Clin Exp Immunol 158: 260-271, 2009.

29. Shahsavan S, Pirayesh A, Samani OZ, Shirzad H, Zamani MA, Amani S, Kazemi SM, Moghni M, Deris F, Bageri N, et al: The relationship between IL-17A and IL-22 expression and clinical severity in patients with moderate/severe persistent allergic rhinitis. Am J Otolaryngol 40: 173-178, 2019.

30. Rosenberg HF, Phipps S and Foster PS: Eosinophil trafficking in allergy and asthma. J Allergy Clin Immunol 119: 1303-1310, quiz 1311-1312, 2007.

31. Wills-Karp M, Luyimbazi J, Xu X, Schofield B, Neben TY, Karp CL and Donaldson DD: Interleukin-13: Central mediator of allergic asthma. Science 282: 2258-2261, 1998. 
32. Zhong H, Fan XL, Fang SB, Lin YD, Wen W and Fu QL: Human pluripotent stem cell-derived mesenchymal stem cells prevent chronic allergic airway inflammation via TGF- $\beta 1-S m a d 2 / S m a d 3$ signaling pathway in mice. Mol Immunol 109: 51-57, 2019.

33. Norris GT and Kipnis J: Immune cells and CNS physiology: Microglia and beyond. J Exp Med 216: 60-70, 2019.

34. Rubtsov YP, Rasmussen JP, Chi EY, Fontenot J, Castelli L, Ye X, Treuting P, Siewe L, Roers A, Henderson WR Jr, et al: Regulatory $\mathrm{T}$ cell-derived interleukin-10 limits inflammation at environmental interfaces. Immunity 28: 546-558, 2008.

35. Stockwell BR and Jiang X: A Physiological Function for Ferroptosis in Tumor Suppression by the Immune System. Cell Metab 30: 14-15, 2019.

36. Huang N, Liu K, Liu J, Gao X, Zeng Z, Zhang Y and Chen J: Interleukin-37 alleviates airway inflammation and remodeling in asthma via inhibiting the activation of NF- $\kappa \mathrm{B}$ and STAT3 signalings. Int Immunopharmacol 55: 198-204, 2018.
37. Xuekun H, Qintai Y, Yulian C and Gehua Z: Correlation of gammadelta-T-cells, Th17 cells and IL-17 in peripheral blood of patients with allergic rhinitis. Asian Pac J Allergy Immunol 32: 235-239, 2014.

38. Kidd P: Th1/Th2 balance: The hypothesis, its limitations, and implications for health and disease. Altern Med Rev 8: 223-246, 2003.

39. Zhu J, Dong J, Ji L, Jiang P, Leung TF, Liu D, Ng LG, Tsang MS, Jiao D, Lam CW, et al: Anti-allergic inflammatory activity of interleukin-37 is mediated by novel signaling cascades in human eosinophils. Front Immunol 9: 1445, 2018.

40. Campbell TM and Bryceson YT: IL2RB maintains immune harmony. J Exp Med 216: 1231-1233, 2019.

This work is licensed under a Creative Commons Attribution-NonCommercial-NoDerivatives 4.0 International (CC BY-NC-ND 4.0) License. 